\title{
Advances in optical earth observation for geological mapping: A Review
}

\author{
L. Ngcofe ${ }^{1}$, A. Van Niekerk ${ }^{2}$ \\ ${ }^{1}$ Department of Rural Development and Land Reform. Directorate: National Geo-Spatial \\ Information, Luncedo.Ngcofe@drdlr.gov.za \\ ${ }^{2}$ Department of Geography \& Environmental Studies, Stellenbosch University, \\ avn@sun.ac.za
}

DOI: http://dx.doi.org/10.4314/sajg.v5i1.1

\begin{abstract}
Recent increases in the availability of earth observation (EO) data and the advances made in its processing have opened up new opportunities for earth monitoring studies. This article reviews recent advances in EO, specifically in the optical domain, and comments on their potential for geological investigations. A review of the literature revealed that much work has been done on the use of multispectral to hyperspectral EO data for geological investigations, but that relatively little emphasis has been placed on the value of high spatial resolution in geological studies. From the literature it was found that geographic object based image analysis (GEOBIA) is a relatively new paradigm in remote sensing that has been shown to reduce the intra-class spectral variability in high spatial resolution imagery, thereby increasing image classification accuracies. It has been used for many applications, but relatively little research has been done on the application of GEOBIA for geological investigations. Another finding of this review is that the power of EO data increases substantially when different types of data are used (fused) together. Data fusion seizes the advantages of the capabilities of each sensor while reducing its limitations. By its nature, GEOBIA offers the ability to fuse disparate data sources and the review concludes that more research is required on the use of GEOBIA and data fusion of high resolution EO data for geological mapping.
\end{abstract}

\section{Introduction}

Remote sensing (RS) of the earth, or earth observation (EO), can be defined as the science of the identification of earth-surface features and the estimation of their geophysical and biophysical properties using electromagnetic radiation, measured by sensors on airborne (aircraft) or spaceborne (satellites, space shuttles) platforms (Agar and Coulter, 2007; Drury, 2001). EO has played an important role in geological mapping, especially in areas with limited infrastructure and harsh environmental conditions. EO methods offer: the opportunity to analyse and map surface geology in a relatively short time and at reduced cost; provide additional (sometimes new) information for preliminary geological investigations; and give a synoptic view of a study area often difficult to obtain from field-based observation alone (Chernicoff and Nash, 2002; Kariuki et al., 2004; Salati et al., 2011). Broad lithological information is deduced 
from a variety of indicators observed in remotely sensed imagery, including landforms, structural features, soils, vegetation types and drainage patterns (Agar and Coulter, 2007; Gad and Kusky, 2006; 2007; Hewson et al., 2005; Rajesh, 2004). Many authors have studied the strong correlation between lineament interpretation and analysis of remotely sensed imagery, leading to discoveries of major mineral deposits of great economic value (Fernades da Silva et al., 2005; Hashim et al., 2013; Meijerink, 1996; Rajesh, 2004; Rinaldi, 2007; Saadi et al., 2011). Mineral exploration often employs EO to map fault fractures because of their known geological and spatial association with ore deposits, together with frequent targeting of indicator minerals, which often highlight the alteration mechanisms of economic mineral deposits (Azizi et al., 2010; Gabr et al., 2010; Pour and Hashim 2011a; 2011b; Sabins, 1999). A number of useful reviews of EO applications are available (Agar and Coulter, 2007; Bhan and Krishnanunni, 1983; Goetz, 2009; Koirala, 1997; Lipton 1997; Navalgund et al., 2007; Rajesh, 2004; Sabins, 1999; Van der Meer et al., 2012). This paper expands on the existing reviews by considering recent developments in RS which could potentially aid geological investigations. The focus will be on improvements in spatial and spectral resolution of optical imagery and the value of data fusion for geological applications.

\section{Overview of EO techniques}

The application of EO has increased tremendously since its development in the early nineteenth century. Its capability to measure electromagnetic energy in the visible short-wave infrared (VSIR), thermal infrared (TIR) and microwave wavelength bands has led to the application of EO to geological investigations. Earth surface materials can be remotely detected by studying the way in which they reflect wavelengths with different intensities (Drury, 2001; Goetz, 2009; Sabins, 1999).

An EO sensor's ability to record the reflected intensities is based on its spectral and spatial resolution features. The spectral resolution of a sensor is defined by the number of spectral bands - ranging from a few (multispectral) to hundreds (hyperspectral) - a sensor can record. Spatial resolution denotes the smallest area of the ground surface for which electromagnetic energy can be recorded. Figure 1 provides an overview of some of the prominent EO sensors and their respective spectral ranges used for various geological investigations and other earth monitoring studies. 


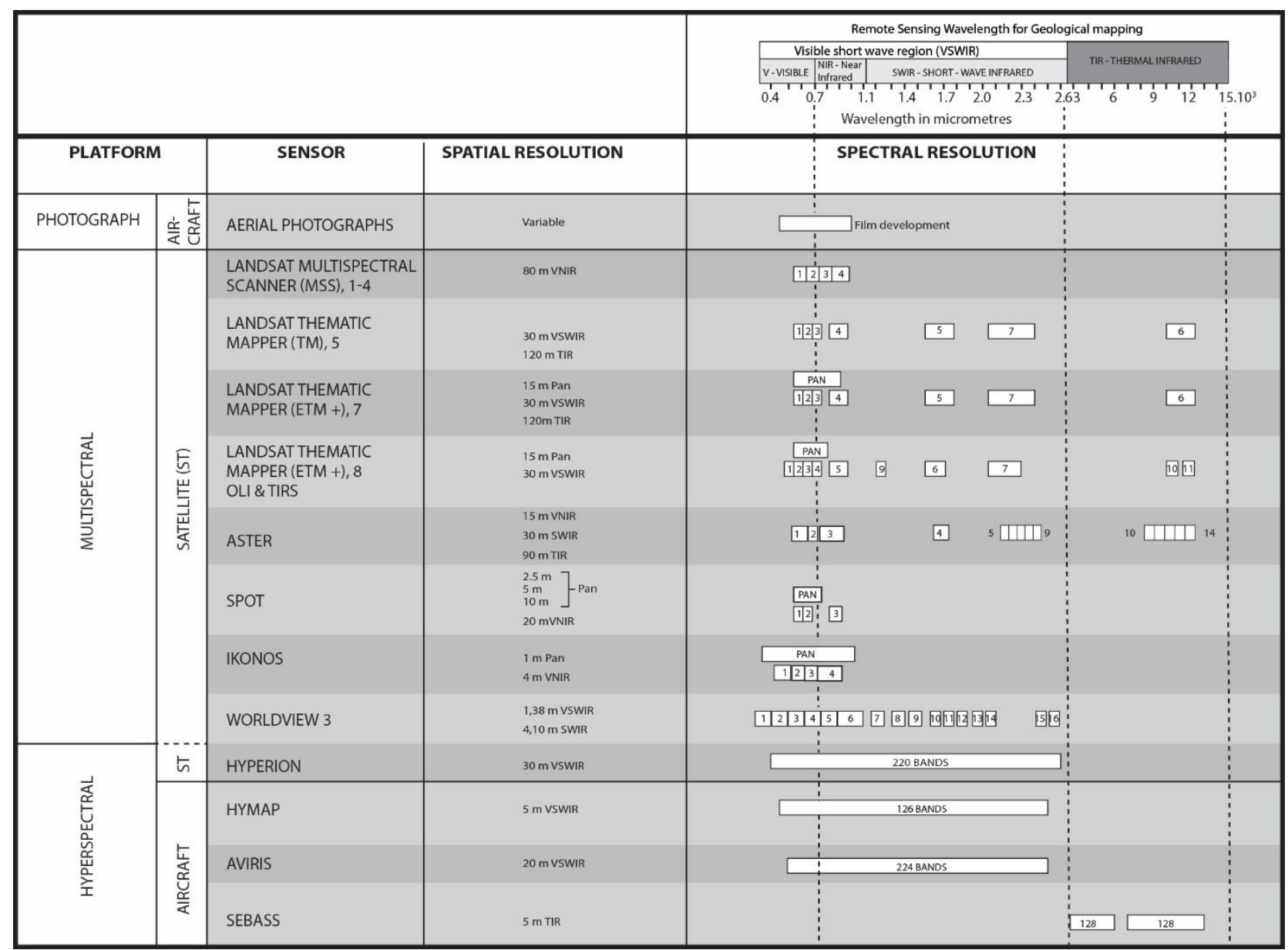

Figure 1. Optical earth observation sensors with their spectral and spatial resolution characteristics (adapted from Mauger, 2003)

The required spectral and spatial resolutions depend on the application. A high spatial resolution, for instance, is often favoured in urban monitoring applications, while a high spectral resolution is often of more value in geological applications (Drury, 2001; Goetz, 2009; Sabins, 1999; Van der Meer, et al., 2012). High spectral resolution is needed in many geological applications since they rely on the detection of unique spectral signatures of different rock forming minerals (Figure 2), caused by electronic transitions and ion vibrations of such minerals (Drury, 2001; Kruse and Lefkoff, 1993; Navalgund et al., 2007; Rajesh, 2004; Sabins, 1999). Electronic transitions are most often created by iron, while vibrational transitions are often created by water, hydroxyl ions and carbonates (Rowan et al., 2004). The absorption feature indicating iron oxide electronic transitions is reflected by wavelengths in the range of 0.35 to $1.5 \mu \mathrm{m}$, while charge-transfer transitions are apparent at wavelengths shorter than $0.55 \mu \mathrm{m}$. Vibration transitions produce reflectance anomalies in the near-infrared region of the spectrum between $1.1 \mu \mathrm{m}$ and $2.5 \mu \mathrm{m}$, while $\mathrm{Mg}-\mathrm{OH}$ also produces distinctive absorption features in this range. Vibration transitions associated with carbonates yield absorption features at about $2.3 \mu \mathrm{m}$ 
(Drury, 2001; Rajesh, 2004).

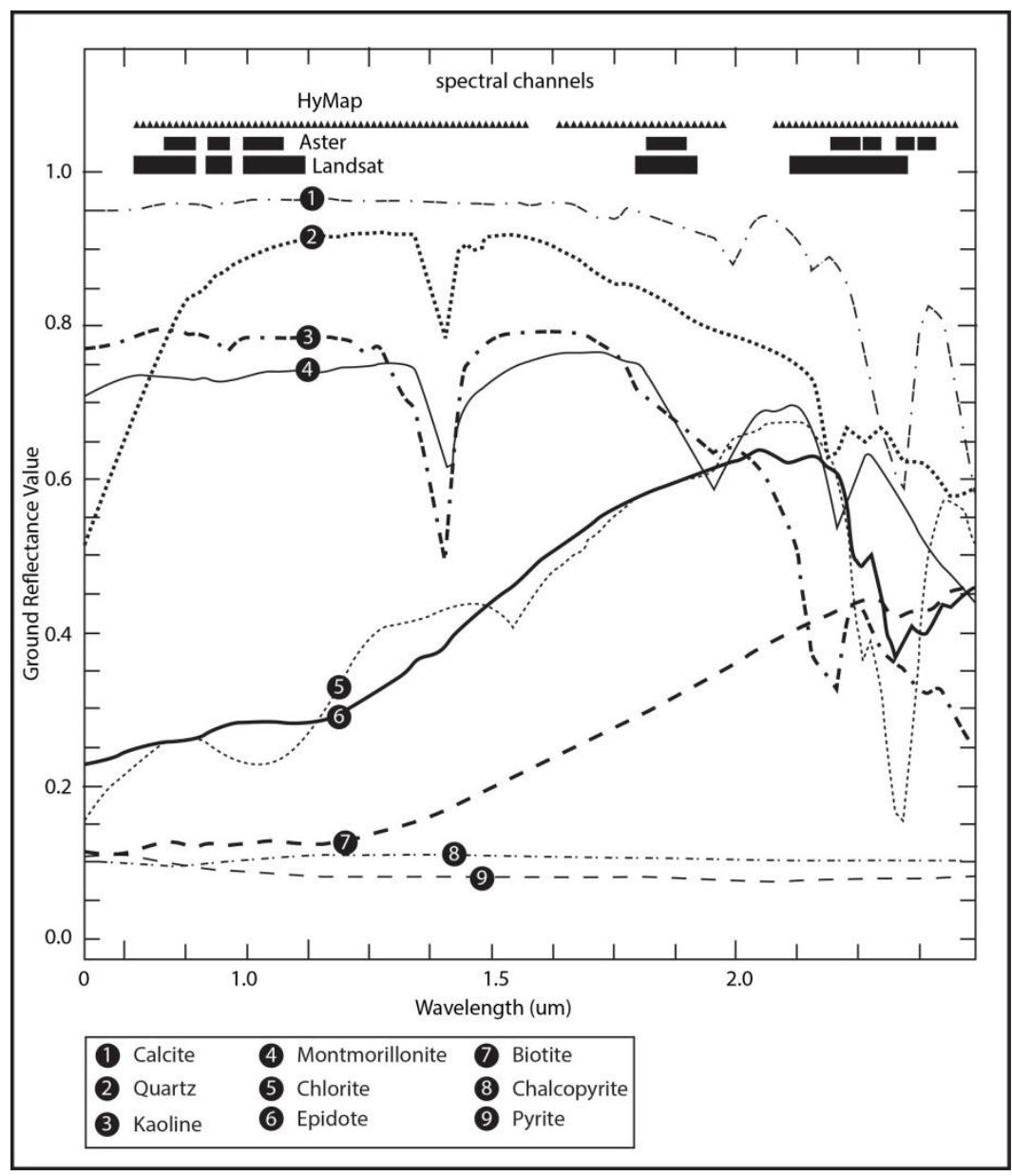

Figure 2. Mineral spectral signatures of some common rock forming minerals (adapted from Cocks et al., 1998)

Various techniques for the analysis of EO imagery for geological investigations are available. Of these the most popular approaches are band ratios (Assiri et al., 2008; Drury, 2001; Gabr et al., 2010; Pour and Hashim, 2012a; 2012b); principal component analysis (PCA) (Gad and Kusky, 2007; Lipton, 1997; Rajesh, 2008; Sabins, 1999) and image classification (Brogaard and Prieler, 1998; Campbell, 1996; Gibson and Power, 2000).

The application of TIR EO data for geological investigations is popular owing to its ability to record the earth's surface emissivity (or temperature) and the rate of heat loss of different surface materials, which often indicates geophysical properties. TIR images are usually recorded in the range of 3 to $15 \mu \mathrm{m}$, although the absorption by atmospheric gases restricts thermal sensing in the regions of 3 to $5 \mu \mathrm{m}$ and 8 to $14 \mu \mathrm{m}$ (Drury, 2001; Rowan and Mars, 2003; Van der Meer et al., 2012; Vaughan et al., 2003). According to Hecker (2006), TIR data complements the information provided by VSIR, particularly in geological studies where the distribution of 
silicate minerals is important. The absorption features of silicate minerals are observed around 8 to $12 \mu \mathrm{m}$ wavelength thus making TIR systems invaluable for silicate mineral detection.

\section{Advances in EO image processing}

The recent advances in EO have concentrated on the development and processing of imaging spectrometry, also known as hyperspectral imaging. The development of hyperspectral imaging has been documented by several authors (Cloutis, 1996; Goetz, 2009; Kruse and Lefkoff, 1993; Plaza et al., 2009). The acquisition capability of hundreds of narrow contiguous spectral bands enhances the identification of earth-surface materials by their characteristic reflectance or absorption discriminatory features. An example of such a sensor is the airborne visible infrared imaging spectrometer (AVIRIS), developed in 1984 with 224 contiguous spectral bands from 4.0 to $2.5 \mu \mathrm{m}$ at $20 \mathrm{~m}$ spatial resolution. The HyMap hyperspectral imaging sensor, developed by the Australian company HyVista (Figure 1), is another example. The sensor covers the 0.45 to $2.5 \mu \mathrm{m}$ wavelength region in 126 spectral bands at $5 \mathrm{~m}$ spatial resolution (Cocks et al., 1998). Both the AVIRIS and HyMap operate on an aircraft platform. Currently, the only earthorbiting hyperspectral imaging sensor is Hyperion on the Earth Observation-1 (EO-1) satellite which was launched in the year 2000. It has the ability to acquire data in 220 spectral bands from 0.356 to $2.5 \mu \mathrm{m}$ at $30 \mathrm{~m}$ spatial resolution (Kruse et al., 2003).

The advent of high spectral resolution sensors led to the development of a suite of techniques for extracting spectral information, the goal being the derivation of surface compositional information of the earth's surface (Cloutis, 1996; Van der Meer et al., 2012). Two broad categories of analytical techniques were developed and widely used for classifying hyperspectral data, namely spectrum-matching techniques and subpixel unmixing techniques. Spectrum-matching techniques express the spectral similarity of reference (derived from spectral library or field spectra of known materials) to test (image) spectra. This technique is applied through the spectral angle mapper (SAM) which measures spectral similarities by calculating the angle between the reference spectra and the test spectra (Bertels et al., 2005; Kruse 1998; Van der Meer, 2006). The subpixel unmixing techniques aim to quantify the relative abundance (fractions, percent or area) of various materials within a pixel. This is based on the premise that a pixel may represent multiple surface materials, resulting in mixed (averaged) spectral signatures. Subpixel unmixing techniques separate such average spectral signatures into pure spectral 
signatures which represent each material covered by the pixel.

The spatial resolution is also vital as it determines which earth-surface objects are detectable (Yu et al., 2006). Finer (or higher) spatial resolution images provide greater spatial detail and therefore enable the delineation of small features compared to coarse (or low) spatial resolution images (Aplin, 2006; Mather, 2003; Woodcock and Strahler, 1987). Consequently, the spatial resolution of an image directly influences the scale at which it can be applied. The gradual refinement of geological work from scales of 1:250 000 to $1: 50000$, and even 1:10 000 to 1:5 000, requires EO images with increasing spatial resolutions. For instance, early applications of EO to geological applications made use of Landsat (MSS) imagery with 80m resolution (Agar and Coulter, 2007; Bhan and Krishnanunni, 1983). The availability of 30m resolution Landsat (TM) imagery has enabled larger scale mapping (Gad and Kusky, 2006; Mohammad et al., 2001), while 5m resolution HyMap data is suitable for even larger scale surveys (Cloutis, 1996; Cocks et al., 1998; Yu et al., 2006).

Current advances in EO are providing images with increasing spatial resolutions of up to $31 \mathrm{~cm}$ (World View 3 panchromatic band, launched on the $13^{\text {th }}$ of August 2014). However, the inherent problem with the use of high spatial resolution imagery is its increase in intraclass spectral variability within homogenous earth-surface features (e.g. land-cover types) which decreases the statistical separability of such features in spectral data space and subsequently reduces the accuracy of image classifications (Aplin et al., 1997; Carleer et al., 2005; Woodcock and Strahler, 1987, Yu et al., 2006). To overcome the intraspectral variability caused by improved spatial resolution, geographic (also known as geospatial) object-based image analysis (GEOBIA) has been proposed (Blaschke, 2010; Blaschke and Hay, 2001; Hay and Castilla, 2008). GEOBIA involves partitioning a scene into discrete entities or segments from which meaningful image objects, based on their spatial and spectral attributes, can be generated (Desclee et al., 2006; Kim et al., 2009). The basic assumption of GEOBIA is that the image being classified is made up of relatively homogenous patches that are larger than individual pixels. To analyse EO images, GEOBIA starts by grouping pixels into image-objects (Hay and Castilla, 2008; Chen et al., 2008; Darwish et al., 2003; Orkonselenge, 2004) called image segmentation, a fundamental step in GEOBIA.

Image segmentation diminishes the within-object variations, while emphasizing the inter-object variations (Benz et al., 2004; Blaschke, 2010). This is achieved by the 
merging of pixels into objects and is based on a local homogeneity criterion which defines similarities between adjacent pixels following a combination of colour (spectral value) and spatial properties (size, texture and shape). Various segmentation algorithms are available in literature, with Mishra et al. (2011) categorizing them (segmentation) into four types, namely edge-based, cluster-based, region-based and split-merge approaches. In edge-based approaches an image is partitioned based on abrupt changes in intensity. Image edges are detected and linked into contours that represent boundaries of image objects. In cluster-based approaches image segmentation stores image pixels in ascending order of intensity. A predefined number of clusters are separated by using an intensity histogram. In region-based approaches, the segments with similar intensities are merged according to a decision rule, while split/merge approaches are based on a colour and texture algorithm.

\section{Examples of EO in geological applications}

Notable publications in which EO was used for geological applications are summarized in this review. Chernicoff and Nash (2002) combined a 1-4-7 composite of Landsat Thematic Mapper (TM) bands with aeromagnetic data for analysing geological structures in the northern Precordillera region of Argentina. The information derived from the Landsat (TM) imagery conformed to published maps and also revealed a new set of previously unknown structures. The resulting structures were superimposed on an aeromagnetic image, which enabled the precise delineation of lithomagnetic domain boundaries and magnetic pattern breaks (inferred faults). The combination of these datasets led to the definition of a major structural system which controls the economic potential of gold targets in the study area. Hewson et al. (2005) used ASTER data to generate new, accurate and seamless geological and mineralogical information in Australia. Major minerals rich in $\mathrm{Al}-\mathrm{OH}, \mathrm{Mg}-\mathrm{OH}$, carbonates and quartz abundances were targeted. Several band ratios were applied to the target minerals and the results were bound to be satisfactory when compared to those of a HyMap reference survey. ASTER data was also used by Gad and Kusky (2007) for mapping lithology in Egypt. A 7-3-1 band combination was effective for distinguishing between granitic rocks and metamorphic rocks, while other band ratios successfully discriminated between vast rock units (gneiss, migmatite, amphibolite), volcanogenic sediments with banded iron formation, metabasalt and granitic rocks. The maps produced using ASTER data were 
integrated with field observations to produce an enhanced geological map. In Namibia Gomez et al. (2005) successfully applied PCA to ASTER data in the visible near infrared (VNIR) and short-wave infrared (SWIR) spectra to improve geological mapping. Of the nine PCA components generated, the first five represented the most (98\%) information, while the last four components were less useful as it contained abundant noise (Gomez et $a l ., 2005)$. The results provided new lithological boundary information that was used to upgrade the geological map of the area.

HyMap data was applied to map the Sarfartoq carbonatite complex in south-west Greenland (Bedini, 2009). The minimum noise fraction (MNF) transformation, a spectral reduction and data compression technique, was used. MNF is a form of PCA but instead of ordering the data by variance, the data is ordered according to a signal-to-noise ratio (Bertels et al., 2005; Chen, 2000). The first $20 \mathrm{MNF}$ bands were used as input to an unsupervised classification algorithm to map carbonatite, carbonatite outer core zones (fenite with carbonatite dyke), fenite, marginal alteration zone (hematized gneiss) and gneiss. The classification achieved an overall accuracy of $87.6 \%$ and improved the geological mapping of the study area's carbonatite complex. HyMap and AVIRIS data were also used to map expansive clay soils in Colorado USA (Chabrillat et al., 2002). Smectite, illite and kaolinite were the targeted clay minerals. These clay minerals were identified by using higher-order MNF bands focusing on 2.0 to $2.45 \mu \mathrm{m}$ as clay absorbs wavelengths in this region of the spectrum. Field investigations gathered information on mineralogy and the swelling potential of the samples collected was determined by standard laboratory analyses. Together with EO images, maps of clay types with potential swelling were successfully compiled.

For many years EO for geological mapping was focused on the use of spectral information to detect alteration zones (Pour and Hashim, 2012a; 2012b) and for lithological mapping (Hewson et al., 2005) without exploiting spatial information. It has been acknowledged that the incorporation of spatial information improves spectral classification (Blaschke, 2010), more so in geology due to its ability to infer geological transitions. Traditional EO lithological boundary detection is often subjective, with distinct variation occurring between expert analyses. Saliti et al. (2011) investigated the automatic detection of lithological boundaries based on the pattern rotational variant template edge matching algorithm of ASTER imagery in the South West of Iran. The accuracy of the detected lithological boundaries was spatially assessed by five experts against two reference sources: a published geological map produced by Iranian oil operation 
companies (scale 1:100 000) and boundaries interpreted from a pair of stereo aerial photographs (scale 1:50 000). Although the results could not be quantified (due to no ground truth data of mineral composition), a comparison of the boundary detection with those of reference sources showed a spatial correspondence between rotational variant template edge matching technique and reference boundaries. It was noted that in heterogeneous areas this method did not correlate with the reference boundaries. Saha et al. (2011) applied an object-based approach to map drumlin landforms from 30m digital elevation model (DEM) in the Chautauqua drumlin field in North West Pennsylvania and New York. The shape and orientation of drumlin provides a wealth of information about the flow of past glaciers. Through the RGB combination of DEM, slope and aspect, drumlins were manually digitised by an expert and compared to the drumlins derived through automated GEOBIA multiresolution image segmentation algorithm. Using the drumlins which were manually digitised by an expert as a reference, the drumlins from automated method were visually and statistically compared to the reference drumlins. The results revealed that the automated procedure had over-identified the drumlins. Some of the over-identified drumlins were actually not drumlins, while other drumlins were underclassified by the manual method. The shape and length analysis of the drumlins that were comparable (overlaying each other) after the two methods (manual and automated) were applied, produced varied results, with the automated procedure having greater length values than the manual method. The need for focused research in GEOBIA, specifically for geological applications, can enhance the objectivity of results conducted by different expects.

\section{EO image data fusion}

Advances in sensor technology have significantly improved the spatial and spectral resolution coverage (from multispectral to hyperspectral) of remotely sensed data. Due to their respective capabilities and limitations, the different data types provide different representations of the same physical environment. When analysing an area it is desirable to use all the available data to increase the extraction of information and to improve the accuracy of image classifications (Zhang, 2010). To take full advantage of the increasing availability of EO data, image data fusion has been proposed (Houzelle and Giraudon, 1994; Khaleghi et al., 2013; Maitre and Bloch, 1997; Pohl and Van Genderen, 1998; Simone et al., 2002; Zhang, 2010). Image data fusion takes advantage of the capabilities 
of each sensor and reduces its limitations. Acknowledging this, Khaleghi et al. (2013) maintain that the use of different sensors (or EO images) of the same scene provides a better understanding of the physical properties of the observed objects. Remotely sensed image data fusion is the integration of information acquired at different spatial and spectral resolutions from the same or different sensor platforms (e.g. satellite or aircraft) to produce fused data that contain more detailed information than a single image (Pohl and Van Genderen, 1998). Its advantage is the robust operational performance it provides, i.e. increased confidence, reduced ambiguity, improved detection and reliability, as well as enhanced classification ability (Du et al., 2013; Khaleghi et al., 2013; Pohl and Van Genderen, 1998; Zhang, 2010). EO data fusion can be performed on pixel/data, feature or decision levels. Pixel/data level fusion combines the original data from multiple sources into single-resolution data, whereas in feature level fusion the features of interest are extracted separately from the data and then combined into singlefeature datasets that may be processed further. GEOBIA has the unique ability to fuse disparate data sources based on groupings of pixels (objects), a capability that is not well researched, particularly in the geological domain.

The geological investigations such as the detection of potential faults, landslides and rock-fall have been conducted during the world's largest water conservancy project in China (Yang et al., 2000). Through the data fusion of SPOT panchromatic (2.5m) band together with SPOT multiresolution $(10 \mathrm{~m})$ bands, a high spatial quality transformation of multispectral content was obtained. The second image fusion in this study was conducted with the enhanced SPOT multispectral fused with synthetic aperture radar (SAR) imagery. With the ability to detect sub-surface structures, together with high spatial multispectral resolution, the results from the image fusion improved the interpretation of linear structures in the study area. Pournamdari and Hashim (2014) also applied data image fusion of ASTER and Landsat TM for geological mapping of an ophiolite complex (the source of chromite ore deposits in the area) in South East Iran. A feature level fusion technique was applied on spectral angle mapper results derived from ASTER and Landsat imagery respectively to successfully derive a lithological map of the ophiolite complex.

Although data fusion enhances geological investigations, challenges still remain. The data imperfection, correlation, inconsistency and disparateness due to the diversity of the sensor technologies are among the data fusion glitches (Khaleghi et al., 2013). While investigations have been conducted into data fusion algorithms (Du et al., 2013; Pohl and 
Van Genderen, 1998), no single data fusion algorithm is as yet able to overcome all the obstacles of data fusion. Research on data fusion suitable for geological investigations would eliminate inaccuracies resulting from the incorrect application of data fusion algorithms.

\section{Conclusion}

Technological developments in EO significantly influenced geological investigations. Recent improvements in sensor technology and the availability of higher spatial and spectral resolution data hold great potential for geological studies. EO techniques can be used to derive new information about the reflectance of rocks which can be employed for very accurate delineation of lithological formations. In some studies like urban monitoring applications, the challenges posed by increases in the intraclass spectra variability, triggered by increasing spatial resolution of imagery, can be addressed by the application of GEOBIA. More research on the use of GEOBIA for geological applications is urgently required. The recent launch of Sentinel 1a in April 2014, together with planned launch (in 2016) of Sentinel 1b RADAR imaging mission, provide potential for data fusion application with already launched Landsat 8 (February 2013), Worldview 3 (August 2014) and recently launched Sentinel 2a (and planned 2b) passive satellites. However, a number of challenges with data fusion have been noted. Most of these challenges arise from the data to be fused, imperfections and diversity of the sensor technologies, and the nature of the application environment (Houzelle and Giraudon, 1994; Khaleghi et al., 2013). A better understanding of these issues and the implementation of controlling measures will increase the potential of EO for geological applications. New technologies, such as GEOBIA, also offer potential solutions but more work is required to understand its value and implementation.

\section{References}

Agar, B. \& Coulter, D. 2007. 'Remote sensing for mineral exploration - A decade perspective 1997-2007', in B Milkereit (ed.), Proceedings of Exploration 07: Fifth Decennial International Conference of Mineral Exploration; 109-136.

Aplin, P. 2006. On scale and dynamics in observing the environment. International Journal of Remote Sensing, 27: 2123-2140.

Aplin, P., Atkinson, P.M. \& Curran, P.J. 1997. Fine spatial resolution satellite sensors for the next decade. International Journal of Remote Sensing, 18: 3873-3881. 
Assiri, A., Alsaleh, A. \& Mousa, H. 2008. Exploration of hydrothermal alteration zones using ASTER imagery: A case study of Nuqrah Area, Saudi-Arabia. Asian Journal of Earth Sciences, 2 (1): 77-82.

Azizi, H., Tarverdi, M.A. \& Akbarpour, A. 2010. Extraction of hydrothermal alterations from ASTER SWIR data from east Zanjan, northern Iran. Advances in Space Research 46: 99-109.

Bedini, E. 2009. Mapping lithology of the Sarfartoq carbonatite complex, southern West Greenland, using HyMap imaging spectrometer data. Remote Sensing of Environment, 113: 1208-1219.

Bedini, E. 2011. Mineral mapping in the Kap Simpson complex, central East Greenland, using HyMap and ASTER remote sensing data. Advances in Space Research, 47: 60-73.

Benz, U.C., Hofmann, P., Willhauck, G., Lingenfelder, I. \& Heynen, M. 2004. Multiresolution, object-oriented fuzzy analysis of remote sensing data for GIS-ready information. ISPRS Journal of Photogrammetry \& Remote Sensing, 58: 239-258.

Bertels, L., Deronde, B., Kempeneers, P., Provoost, S. \& Tortelboom, E. 2005. Potential of airborne hyperspectral remote sensing for vegetation mapping of spatial heterogeneous dynamic dunes: A case study along the Belgian coastline. International Conference on Nature Restoration Practices in European Coastal Habitats, Koksijde, Belgium, 153-163.

Bhan, S.K. \& Krishnanunni, K. 1983. Applications of remote sensing techniques to geology. Proceedings of the Indian Academy of Science. Engineering Sciences, 4 (6): 297311.

Blaschke, T. 2010. Object based image analysis for remote sensing. ISPRS Journal of Photogrammetry and Remote Sensing, 62: 2-16.

Blaschke, T. \& Hay, G.J. 2001. Object-oriented image analysis and scale-space: Theory and methods for modelling and evaluating multiscale landscape structure. International Archives of Photogrammetry and Remote Sensing, 4 (34): 22-29.

Brogaard, S. \& Prieler, S. 1998. Land cover in the Horqin Grassland, North China. Detecting changes between 1975 and 1990 by means of remote sensing. International Institute for Applied Systems Analysis; Interim Report IR-98-044/July.

Campbell, J.B. 1996. Introduction to remote sensing 2nd edn. Taylor and Francis Ltd London.

Carleer, A.P., Debeir, O. \& Wolff, E. 2005. Assessment of very high spatial resolution satellite image segmentations. Photogrammetric Engineering and Remote Sensing, 11 (71): 1285-1294.

Chabrillat, S., Goetz, F.H., Krosley, L. \& Olsen, H.W. 2002. Use of hyperspectral images in the identification and mapping of expansive clay soils and the role of spatial resolution. Remote Sensing of Environment, 82: 431-445.

Chen, C. 2000. Comparison of principal component analysis and minimum noise fraction transformation for reducing the dimensionality of hyperspectral imagery. Journal of Geographic Research, 33: 163-178.

Chen, J., Luo, M., Li, L., Li, D., Zhang, C., Huang,Y. \& Jiang, Y. 2008. Comparation and analysis methods of moderate resolution satellite remote sensing image classification. WSEAS Transactions on Computers, 7 (7): 877- 886.

Chernicoff, C.J. \& Nash, C.R. 2002. Geological interpretation of Landsat TM imagery and aeromagnetic survey data, northern Precordillera region, Argentina. Journal of South American Earth Sciences, 14: 813-820.

Christensen, P.R., Bandfield, J.L., Hamilton, V.E., Howard, D.A., Lane, M.D., Piatek, J.L., Ruff, S.W. \& Stefanow, W.L. 2000. A thermal emission spectral library of rockforming minerals. Journal of Geophysical Research, 4 (105): 9735-9739.

Cloutis, E.A. 1996. Hyperspectral geological remote sensing: Evaluation of analytical techniques. International Journal of Remote Sensing, 12 (17): 2215-2242. 
Cocks, T., Jenssen, R., Stewart, A., Wilson, I. \& Shield, T. 1998. The HyMap airborne hyperspectral sensor: The system calibration and performance. 1st EARSEL Workshop on Imaging Spectroscopy, Zurich, Swirtzerland, 37-42.

Darwish, A., Leukert, K. \& Reinhardt, W. 2003. Image segmentation for the purpose of object based classification. IEEE, 3: 2039-2041.

Desclee, B., Bogaert, P. \& Defourny, P. 2006. Forest change detection by statistical object-based method. Remote Sensing of Environment, 102: 1-11.

Drury, S. 2001. Image Interpretation in geology, $3^{\text {rd }}$ Ed. Blackwell Science Inc, Malden.

Du, P., Lui, S., Xia, J. \& Zhao, Y. 2013. Information fusion techniques for change detection from multi- temporal remote sensing images. Information Fusion, 14: 19-27.

Ellis, R.J. \& Scott, P.W. 2004. Evaluation of hyperspectral remote sensing as a means of environmental monitoring in the St. Austell China clay (kaolin) region, Cornwall, UK. Remote Sensing of Environment, 93: 118-130.

Fernandes da Silva, P.C., Cripps, J.C. \& Wise, S.M. 2005. The use of remote sensing techniques and empirical tectonic models for inference of geological structures: Bridging from regional to local scales. Remote Sensing of Environment, 96: 18-36.

Gabr, S., Ghulam, A. \& Kusky, T.M. 2010. Detecting areas of high-potential gold mineralisation using ASTER data. Ore Geology Reviews, 38: 59-69.

Gad, S., Kusky, T. 2006. Lithological mapping in the eastern desert of Egypt, the Barramiya area, using Landsat thematic mapper (TM). Journal of African Earth Sciences, 44: 196-202.

Gad, S. \& Kusky, T. 2007. ASTER spectral ratioing for lithological mapping in the Arabian-Nubian shield, the Neoproterozoic Wadi Kid area, Sinai, Egypt. Gondwana Research, 11: 326-335.

Gibson, P.J. \& Power, C.H. 2000. Introductory remote sensing: Digital image processing and applications. Taylor and Francis New York.

Goetz, A.F.H. 2009. Three decades of hyperspectral remote sensing of the earth: A personal view. Remote Sensing of Environment, 1 (113): 5-16.

Gomez, C., Delacourt, C., Allemand, P., Ledru, P. \& Wackerle, R. 2005. Using ASTER remote sensing data set for geological mapping in Namibia. Physics and Chemistry of the Earth, 30: 97-108.

Hashim, M., Ahmad, S., Jofari, M.A. \& Pour, A.B. 2013. Automatic lineament extraction in heavy vegetated region using Landsat Enhanced Thematic Mapper (ETM+) imagery. Advances in Space Research, 51: 874-890.

Hay, G.J. \& Castilla, G. 2008. Geographic Object-Based Image Analysis (GEOBIA): A new name for a new discipline. In: Object-based image analysis: Spatial concept for knowledge-driven remote sensing applications edited by Blascke $\mathrm{T}$ and Lang S. (Springer Publishers, Heidelberg), 93-112.

Hecker, C.A. 2006. Geologic surface compositional mapping from thermal infrared SEBASS data. ISPRS mid-term symposium 2006 remote sensing: from pixels to processes, 8-11 May, Enschede, Netherlands.

Hecker, C., Van der Meijde, M. \& Van der Meer F.D. 2010. Thermal infrared spectroscopy on feldspars - Successes, limitations and their implication for remote sensing. Earth Science Reviews, 103: 60-70.

Hewson, R.D., Cudahy, T.J., Mizuhiko, S., Ueda, K. \& Mauger, A.J. 2005. Seamless geological map generation using ASTER in the Broken Hill-Curnamona province of Australia. Remote Sensing of Environment, 99: 159-172.

Houzelle, S. \& Giraudon, G. 1994. Contribution to multisensory fusion formalisation. Robotics and Autonomous System, 13: 69-85.

Kariuki, P.C., Woldai, T. \& Van der Meer, F. 2004. The role of remote sensing in mapping swelling soils. Asian Journal of Geo-informatics, 5: 43-53. 
Khaleghi, B., Khamis, A., Karray, F.O. \& Razavi, S. 2013. Multisensor data fusion: A review of the state-of-the-art. Information Fusion, 14: 28-44.

Kim, M., Madden, M. \& Warner, T.A. 2009. Forest type mapping using object-specific texture measures from multispectral Ikonos imagery: Segmentation quality and image classification issues. Photogrammetric Engineering and Remote Sensing, 7 (75): 819-829.

Koirala, H.L. 1997. Introduction to remote sensing and image interpretation. Tribhuvan University Journal, 1 (10): 109-118.

Kruse, F.A. 1998. Advances in hyperspectral remote sensing for geologic mapping and exploration. In Proceedings $9^{\text {th }}$ Australasian remote sensing conference, Sydney, Australia.

Kruse, F.A. \& Lefkoff, A.B. 1993. Knowledge-based geological mapping with imaging spectrometers. Remote Sensing Reviews, 8: 3-28.

Kruse, F.A., Boardman, J.W. \& Huntington, J.F. 2003. Evaluation and validation of EO1 Hyperion for mineral mapping. In: Special Issue: Transactions on Geosciences and Remote Sensing (TGARS) IEEE, 6 (41): 1388-1400.

Kruse, F.A., Perry, S.L. \& Cabellero, A. 2006. District-level mineral survey using airborne hyperspectral data, Los Menucos, Argentina. Annals of Geophysics, 1 (49): 83-92.

Lipton, G. 1997. Spectral and microwave remote sensing: An evolution from small scale regional studies to mineral mapping and ore deposit targeting. In: Proceedings of Exploration 97: Fourth Decennial International Conference on Mineral Exploration edited by Gubins AG. (Prospectors and Developers Association of Canada), 43-58.

Maitre, H. \& Bloch, I. 1997. Image fusion. Vistas in Astronomy, 3 (41): 329-335.

Mather, P.M. 2003. Computer processing of remote sensed images: An introduction $2^{\text {nd }}$ edn. John Wiley and Sons Inc, New York.

Mauger, A.J. 2003. Comparison of various remote sensing and spectral radiometer instruments. MESA Journal 29: 26-29

Meijerink, A.M.J. 1996. Remote sensing applications to hydrology: Groundwater. Hydrological Sciences Journal, 4 (41): 549-561.

Mishra, P., Srivastava, N. \& Shukla, K.K. 2011. Agent based image segmentation methods: A review. International Journal of Computer Technology and Applications, 3 (2): 704-708.

Mohammad M.R., El-Sobky, H., Sedeik, K. \& El-Raey, M. 2001. Application of band ratios identified by HHRR for recognition of surface units using TM data in Siwa depression, Western Desert, Egypt; Paper presented at the 22nd Asian Conference on Remote Sensing, Singapore.

Navalgund, R.R., Jayaraman, V. \& Roy, P.S. 2007. Remote sensing applications: An overview. Current Science, 12 (93): 1747-1766.

Orkhonselenge, T. 2004. Texture based segmentation of remotely sensed imagery for identification of geological units. Msc thesis, International Institute of Geo-information Science and Earth Observation, Netherlands.

Pena, S.A. \& Abdelsalam, M.G. 2006. Orbital remote sensing for geological mapping in southern Tunisia: Implication for oil and gas exploration. Journal of African Earth Sciences, 44: 203-219.

Plaza, A., Benediktsson, J.A., Boardman, J.W., Brazile, J., Bruzzone, L., Camps-Valls G., Chanussot, J., Fauvel, M., Gamba, P., Gualtieri, A., Marconcini, M., Tilton, J.C. \& Trianni, G. 2009. Recent advances in techniques for hyperspectral image processing. 
Remote Sensing of Environment, 113:10-122.

Pohl, C. \& Van Genderen, J.L. 1998. Multisensor image fusion in remote sensing: concepts, methods and applications. International Journal of Remote Sensing, 5 (19): 823854.

Pour, A.B. \& Hashim, M. 2011a. Application of advanced spaceborne thermal emission and reflection radiometer (ASTER) data in geological mapping. International Journal of the Physical Sciences, 33 (6): 7657-7668.

Pour, A.B. \& Hashim, M. 2011b. Identification of hydrothermal alteration minerals for exploring of porphyry copper deposits using ASTER data, SE Iran. Journal of Asian Earth Sciences, 42: 1309-1323.

Pour, A.B. \& Hashim, M. 2012a. The application of ASTER remote sensing data to porphyry copper and epithermal gold deposits. Ore Geology Reviews, 44: 1-19.

Pour, A.B. \& Hashim, M. 2012b. Identifying areas of high economic-potential copper mineralisation using ASTER data in the Urumieh-Dokhtar Volcanic Belt, Iran. Advances in Space Research, 49: 753-769.

Pournamdari, M. \& Hashim, M. 2014. Feature level fusion for enhanced geological mapping of ophiolite complex using ASTER and Landsat TM data. $8^{\text {th }}$ International Symposium of the Digital Earth (ISDE8), doi:10.1088/1755-1315/18/1/012145.

Rajesh, H.M. 2004. Application of remote sensing and GIS in mineral resource mapping - An overview. Journal of Mineralogical and Petrological Sciences, 99: 83-103.

Rajesh, H.M. 2008. Mapping Proterozoic unconformity related uranium deposits in the Rockhole area, Northern Territory, Australia using landsat ETM+; Ore and Review, 33: 382-396.

Rinaldi, M. 2007. Remote sensing capability in structural geology analysis of different geodynamic settings: The example of Al Qarqaf Arch (Libya). Scientifica Acta, 1 (1): 4346 .

Rowan, L.C. \& Mars, J.C. 2003. Lithological mapping in the Mountain Pass, California area using Advanced Spaceborne Thermal Emission and Reflection Radiometer (ASTER) data. Remote Sensing of Environment, 84: 350-366.

Rowan, L.C., Simpson, C.J. \& Mars, J.C. 2004. Hyperspectral analysis of the ultramafic complex and adjacent lithologies at Mordor, NT, Australia. Remote Sensing of Environment 91: 419-431.

Saadi, N.M., Abdel Zaher, M., El-Baz, F. \& Watanabe, K. 2011. Integrated remote sensing data utilization for investigating structural and tectonic history of the Ghadames Basin, Libya. International Journal of Applied Earth Observation and Geoinformation, 13: 778-791.

Sabins, F.F. 1999. Remote sensing for mineral exploration. Ore Geology Reviews, 14: $157-183$.

Saha, K., Wells, N.A. \& Munro-Stasiuk, M. 2011. An object-oriented approach to automated landform mapping: A case study of drumlins. Computers and Geosciences, 37: 1324-1336.

Saliti, S., Van Ruitenbeek, F.J.A., Van der Meer F.D., Tangestani, M.H. \& Van der Werff, H. 2011. Lithological mapping and fuzzy set theory: Automated extraction of lithological boundary from ASTER imagery by template matching and spatial accuracy 
assessment. International Journal of Applied Earth Observation and Geoinformation, 13: 753-765.

Simone, G., Farina, A., Morabito, F.C., Serpico, S.B. \& Bruzzone, L. 2002. Image fusion techniques for remote sensing applications. Information Fusion, 3: 3-15.

Tessema, A., Nefale, N. \& Sebake, D. 2012. The use of high-resolution airborne magnetic ASTER and Landsat 7 ETM+ images for identification of kimberlite pipes in the northwestern Free State Province, South Africa. International Journal of Remote Sensing, 14 (33): 4356-4373.

Van der Meer, F.D. 2006. The effectiveness of spectral similarity measures for the analysis of hyperpctral imagery. International Journal of Applied Earth Observation and Geoinformation, 8: 3-17.

Van der Meer, F. \& Van der Werff, H.M.A. Van Ruitenbeek, J.A., Hecker, C.A., Bakker, W.H., Noomen, M.F., Van der Meijde, M., Carranza, J.M., de Smeth, B., Woldai, T., 2012. Multi- and hyperspectral geologic remote sensing: An overview. International Journal of Applied Earth Observation and Geoinformation, 1 (14): 112-128.

Vaughan, R.G., Calvin, W.M. \& Taranik, J.V. 2003. SEBASS hyperspectral thermal infrared data: Surface emissivity measurement and mineral mapping. Remote Sensing of Environment, 85: 48-63.

Wester, K., Lunden, B. \& Bax, G. 1990. Analytical processed Landsat TM images for visual geological interpretation in the northern Scandinavian Caledonides. ISPRS Journal of Photogrammetry and Remote Sensing, 45: 442-460.

Woodcock, C.E. \& Strahler, A.H. 1987. The factor of scale in remote sensing. Remote Sensing of Environment, 21: 311-332.

Yang, W., Cauneau, F., Paris, J.P. \& Ranchin, T. 2000. Fusion of SAR and SPOT images for the detection of geological features over the Three Gorges Dam site, China. In $3^{\text {rd }}$ International Conference "Fusion of Earth Data: merging point measurements, raster maps and remotely sensed images. Sophia-Antipolis, France,132-137

Yamaguchi, Y. \& Naito, C. 2003. Spectral indices for lithological discrimination and mapping using the ASTER SWIR bands. International Journal of Remote Sensing, 22 (24): 4311-4323.

Yu, Q., Ging, P., Clinton, N., Biging, G., Kelly, M. \& Schirolauer, D. 2006. Objectbased detailed vegetation classification with airborne high spatial resolution remote sensing imagery. Photogrammetric Engineering and Remote Sensing, 7 (72): 799-811.

Zhang, J. 2010. Multi-source remote sensing data fusion: status and trends. International Journal of Image and Data Fusion 1 (1): 5-24. 